\title{
XLVI. On the shape of movable coils used in electrical measuring-instruments
}

\section{T. Mather}

To cite this article: T. Mather (1890) XLVI. On the shape of movable coils used in electrical measuring-instruments, Philosophical Magazine Series 5, 29:180, 434-439, DOI: $10.1080 / 14786449008619961$

To link to this article: http://dx.doi.org/10.1080/14786449008619961

曲 Published online: 08 May 2009.

Submit your article to this journal $[\pi$

Џll Article views: 2

Q View related articles $\square$ 
facts at his disposal*, yet the hydrates which I mention as existing include those which both Mendeléeff and Crompton mentioned, thus:-

\begin{tabular}{|c|c|c|c|c|c|}
\hline \multicolumn{2}{|c|}{ From Densities. } & \multicolumn{2}{|c|}{ From Conductivities. } & \multicolumn{2}{|c|}{$\begin{array}{l}\text { Mean from all } \\
\text { sources. }\end{array}$} \\
\hline Mendeláeff. & Pickering. & Crompton. & Pickering. & Pic & ng. \\
\hline About 84.5 & At $84 \cdot 48$ & About 84.5 & At $84 \cdot 0$ & $84 \cdot 24$ & cent. \\
\hline$" 73 \cdot 1$ & , 73.07 & $" 731$ & , $73(?)$ & $73 \cdot 04$ & " \\
\hline$" \quad 47 \cdot 6$ & , $50 \cdot 33$ & $\Rightarrow \quad 47 \cdot 6$ & & $49 \cdot 92$ & $"$ \\
\hline & $" 18 \cdot 5$ & $\Rightarrow \quad 18.5$ & & $18 \cdot 92$ & , \\
\hline $\mathbf{3 \cdot 5}$ & $" 4 \cdot 15$ & 3.5 & $" \mathbf{3} 5$ & $3 \cdot 99$ & " \\
\hline
\end{tabular}

I have mentioned above that the second differential is rectilinear in every case; but I do not by any means think that it has been proved to be absolutely so. The investigation was purely experimental in its nature ; and the point of chief importance was that the lines ultimately obtained by differentiation should be straight within the limits of experimental error, for their being so proves that each of them is derived from an independent curve, which, within these sane limits, is regular.

XLVI. On the Shape of Movable Coils used in Electrical Measuring-Instruments. By T. Matrer, Assistant in the Physical Department, Central Institution $\dagger$.

TT is with some diffidence that $I$ venture to bring the sub1 ject of this note before the Society, because it concerns such a fundamental point in the construction of measuringinstruments having movable coils, such as d'Arsonval galvanometers, electro-dynamometers, wattmeters, \&c., that it is almost certain to have been worked out before. However, as the matter is not touched upon in the ordinary text-books, I bring it forward in the hope that it may serve to recall attention to a subject which, judging from the construction of such

* It is not difficult to see how both Mendeléeff and Crompton arrixed at partially right conclusions from erroneous or insufficient premisses (loce. cit. pp. $79,86,125$ ).

$\uparrow$ Communicated by the Physical Society : read March 21, 1890. 
instruments as are in common use, has been almost entirely ignored.

In a paper "On Galvanometers," by Prof. Ayrton, Dr. Sumpner, and the writer, read before the Society on January 17th, it was pointed out that the coils of d'Arsonval galvanometers should be narrow and long, and that there should be no internal core. Mr. C. V. Bors had evidently arrived at the same conclusion long before the date referred to, for the coil of his radio-micrometer is a proof of this.

The object of this paper is to determine the best shape of the section of the coil perpendicular to the axis about which it turns.

The subject will be dealt with as concerning coils suspended in uniform fields, but similar reasoning may be applied to other instruments in which movable coils are used.

Let the point $O$ (fig. 1) represent in plan the axis about which the coil turns, and assume it is placed in a magnetic field whose direction is perpendicular to $\AA \mathrm{B}$.

Let $P$ be an element of the section of the coil, then the deflecting moment exerted by unit length measured at right angles to the paper is

$$
\mathrm{HCar} \sin \theta \text {; . . . . }
$$

where $\mathrm{H}$ is the strength of field, $\mathrm{C}$ the current-density per unit area, $a$ the area of the element, and $r$ its distance from the axis.

Fig. 1.

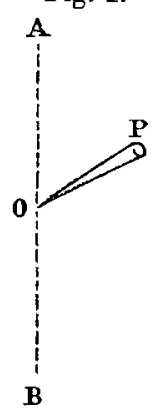

The moment of inertia of the element about $O$ will be

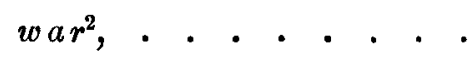

where $w$ is the mass per unit cube.

Now in ordinary commercial instruments it is important that the period of oscillation should not be inconveniently long, and that the power consumed by the instrument should be as small as possible; then, since for a constant period the controlling moment at unit angle must be proportional to the moment of inertia, the problem resolves itself into finding the shape of the section such that the total deflecting moment for a given total moment of inertia is a maximum.

The ratio of the Deflecting Moment to the Moment of Inertia for the element above considered is

$$
\frac{\mathrm{HC} a r \sin \theta}{w a r^{2}} \text {, i. e. :: } \frac{\sin \theta}{r},
$$

since $\mathrm{H}, \mathrm{C}$, and $w$ may be considered constants. 
The ratio $\frac{\sin \theta}{r}$ thus is a measure of the efficacy of the element and its position.

If we now draw a curve whose polar equation is

$$
r=x_{1} \sin \theta,
$$

then, as in Maxwell (vol. ii. p. 332), it may be shown that a given length of wire wound within this space is more efficient than if wound outside it. Hence the curve $r=x_{1} \sin \theta$, i. e. a circle tangential to $A B$ at $O$, is the best form of the section of a movable coil, just as in the case of sensitive galvanometers the best shape is given by

$$
r^{2}=x_{1}^{2} \sin \theta \text {. }
$$

The complete section is given in fig: 2 , and consists of two circles touching at 0 . The circles $C$ and $D$ have section-lines in different directions to indicate that the current passes in opposite directions in the two.

1 The problem may be treated in another way ; for it resolves itself into finding the shape and position of an area having a given moment of inertia about a point in its plane such that the moment of the area about a coplanar line through the point is a maximum.

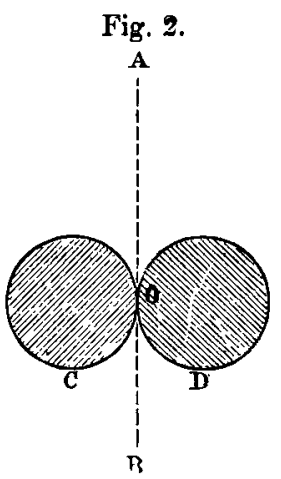

Taking the point as pole, and the line as the line of reference, the expression

whilst

$$
\iint r^{2} \sin \theta d r d \theta \text { is to be a maximum, }
$$

$$
\iint r^{3} d r d \theta \text { is constant. }
$$

This may be solved by the Calculus of Variations with the result above obtained. By this method Dr. Sumpner (to whom the writer suggested the problem) arrived at the solution some time before the author considered the subject from the first point of view.

In order to illustrate the sort of improvement obtained by winding coils to this shape, a table is appended, the first column of which shows various shapes of section, the second column the value of the ratio moment of area $\div$ moment of inertia of area, and the fourth column the deflecting moments of the various shapes all having the unit 
moment of inertia. From this last column it may be seen that the ordinary Siemens dynamometer type of coil, and those of d'Arsonval's galvanometers and wattmeters, are far from being the most efficacious.

The numbers in column 4 are obtained on the assumption that the coils are long in proportion to their breadth; and when this condition is not fulfilled (as it very seldom is), the numbers would be still further reduced, for the parts of the wire at right angles to the axis of suspension add to the moment of inertia but not much to the deflecting force.

This consideration is of importance where the coils are of sections such as (4), (7), (8), (9); for as the coils are wide, the useless moment of inertia sometimes amounts to one fifth the useful. In such a case as (8) the real numbers representing the efficacy of the coil, as compared with one made to section (1) of the same moment of inertia, would be about 1 to 3. These latter numbers represent approximately the efficacy of the coil of an ordinary Siemens dynamometer reading up to 60 amperes, as compared with that of a coil made to the best shape. It is thus possible to increase the deflecting moment for a given current threefold by changing the shape of the movable coil and arranging the fixed coils to produce the same strength of field as at present used.

Of course the new coil would be heavier than the old one, and would therefore require a stronger suspension which might introduce more vagueness of zero ; but as the friction at the mercury-cups is considerable, the necessity for using a stronger suspension would not seriously interfere with the accuracy of the instrument.

It will be noted that in the preceding part of this paper the moment of inertia of the suspended coil has been assumed constant; it is perhaps desirable to give reasons why this assumption is made.

In most instruments having movable coils which are in ordinary use, the current is led into and from the coil either by mercury-cups, or by wires which also serve as the control, or by flexible wires independent of the control.

Taking the first case of mercury-cups, it may be noted that, owing to friction and viscosity, a certain minimum control is necessary to give the requisite definiteness of zero. As time is generally of importance in making commercial measurements, the period of oscillation may be taken as constant. Therefore, as both control and period are determined by circumstances other than those affecting the shape of the coil, the moment of inertia may be taken as constant.

When the current is led into the coil by the torsion-wires, 
the smallest size of the latter is usually determined by considerations of heating, and their length is limited by questions of portability, compactness, and resistance. This means that the control cannot be diminished below a certain minimum, and hence, by reasoning as in the last paragraph, the moment of inertia of the coil must not exceed a certain valne.

The third case, where the leading-in wires are independent of the control, the conditions are somewhat similar to those in the first case ; for the size of these wires is determined by considerations of heating by the current to be measured, and they necessarily possess some viscosity or internal friction, which requires a cortain control to give definiteness of zero.

In addition to the assumption of constant Moment of Inertia, the field in which the coil swings is taken to be uniform and parallel; and consequently the reasoning will not apply to radial fields which exist in instruments such as the new d'Arsonval milliamperemeter, having a range of $180^{\circ}$. In such case it would be advantageous to make the coil of a section similar to that in No. 4 (see table), keeping the radius $b$ as small as possible.

The shape given in No. 1 of table cannot be imitated exactly in practice, for insulation-space is required between the two halves, and unless the coil is used in a zero-instrument, parts near the axis might oppose the rest when a considerable deflexion exists. Shapes (3), (5), and (6) are also open to the same objection, but this is casily surmounted by allowing a small space to exist about the axis ; in fact some such space is almost necessary to enable the coil to he wound conveniently.

Again, in Siemens dynamometers for fairly large currents it would be difficult to make the coil of the best shape, owing to the space required for the mercury-cups being considerable; but a much closer approximation to this shape than the one often employed could be used.

In ordinary d'Arsonvals, which deflect through a considerable angle, the shape should be modified so that no part of it crosses the line A B (fig. 2) even at the maximum deflexion, for any such part would oppose the rest of the coil. The resulting figure would be lemniscate-shaped. There are some cases, however, such as deflexional dynamometers, where it is advisable to make the coil of such a shape as to cross the line A B when deflected; for then the deflexion would increase less rapidly than the square of the current, and hence the instrument would have a greater useful range for a given length of scale. 
used in Electrical Measuring-Instruments.

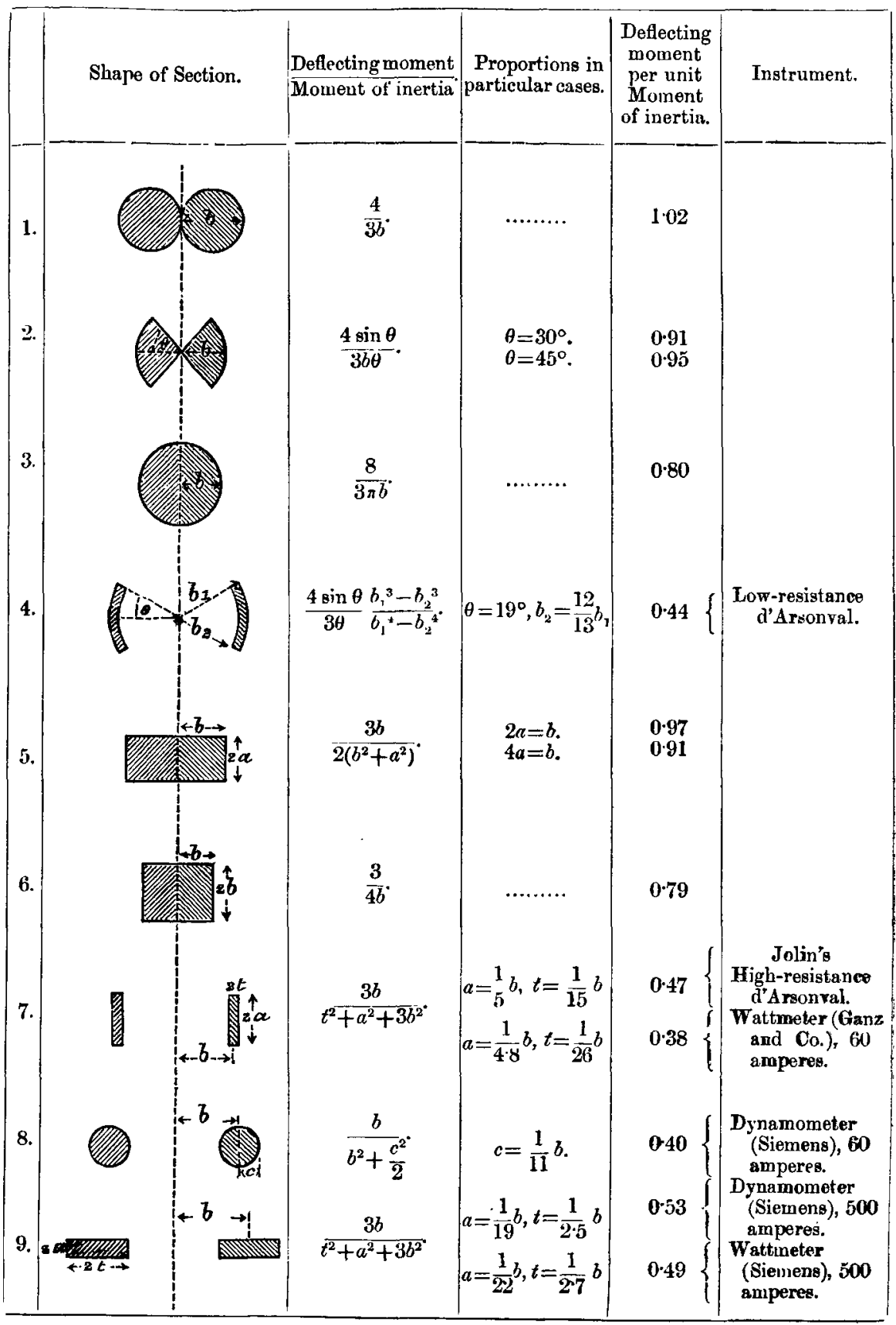

